\title{
Models for the soft X-ray emission of post-outburst classical novae
}

\author{
G. Sala and M. Hernanz
}

\author{
Institut d'Estudis Espacials de Catalunya and Institut de Ciències de l'Espai (CSIC), Campus UAB, Facultat de Ciències, \\ Torre C5-parell, 2a planta, 08193 Bellaterra (Barcelona), Spain \\ e-mail: [sala;hernanz]@ieec.uab.es
}

Received 29 December 2004 / Accepted 12 April 2005

\begin{abstract}
We developed a hydrostatic and stationary white dwarf envelope model for the study of the post-outburst phases of classical novae and their soft X-ray emission. We considered several white dwarf masses and chemical compositions typical of classical novae. Our results show that the luminosity, maximum effective temperature, and envelope masses depend on the white dwarf mass and on the chemical composition. Envelope masses for which equilibrium solutions exist are pretty small $\left(\sim 10^{-7}-10^{-6} M_{\odot}\right)$, thus leading to a short duration of the soft X-ray emitting phase of classical novae, in agreement with most observations. The models we present provide a useful tool for the determination of the white dwarf properties from observable parameters in the $\mathrm{X}$-ray range.
\end{abstract}

Key words. stars: novae, cataclysmic variables - stars: white dwarfs $-\mathrm{X}$-rays: binaries

\section{Introduction}

Classical nova outbursts are caused by explosive burning of hydrogen on the surface of the white dwarf of a cataclysmic variable, a close binary system with mass transfer from a main sequence star (secondary) to a white dwarf (primary). Hydrodynamic models show that, after $10^{-5}-10^{-4} M_{\odot}$ of H-rich material are transferred from the secondary star to the white dwarf, ignition in degenerate conditions takes place in the accreted envelope and a thermonuclear runaway is initiated (Starrfield 1989). As a consequence of the thermonuclear runaway, the envelope expands and causes the brightness of the star to increase by $\sim 10 \mathrm{mag}$, reaching maximum luminosities up to $\sim 10^{5} L_{\odot}$. A fraction of the envelope is ejected at large velocities (hundreds or thousands of $\mathrm{km} \mathrm{s}^{-1}$ ), while a part of it returns to hydrostatic equilibrium with steady nuclear burning. Hydrodynamic models also show that a powerful outburst is only possible if the accreted matter (with solar abundances) is enhanced by some mixing mechanism with $\mathrm{CNO}$ nuclei from the degenerate core. Analyses of nebular emission lines from classical novae indeed show ejecta enhanced in CNO nuclei compared to cosmic abundances and, in some cases, enhancement in $\mathrm{Ne}, \mathrm{Mg}$, and other heavier elements.

The presence of a remaining hydrogen burning envelope on the post-outburst white dwarf was already pointed out after the first hydrodynamic simulations indicated that only a fraction of the hydrogen-rich accreted material is ejected (Starrfield et al. 1974). The rest of the envelope is expected to return to hydrostatic equilibrium with steady hydrogen burning, thereby powering a source of constant bolometric luminosity. As the ejected envelope expands, the photosphere recedes, and inner and hotter layers become visible, shifting the spectrum from optical to ultraviolet and finally to soft X-rays. This picture could explain the persistence of high temperature ultraviolet emission after the optical decline, detected for the first time with OAO-2 in Nova Serpentis 1970 (Gallagher \& Code 1973) and in other novae with IUE (1978-1996).

Without a detailed model for the post-outburst envelope, the duration of the constant bolometric luminosity phase was estimated as the nuclear timescale of the mass left on the white dwarf surface (see for instance Table 1 in Gehrz et al. 1998). Since the outburst ejects a fraction of the accreted envelope, the remaining envelope mass was assumed to be of the same order of magnitude, which was estimated as the envelope mass needed to achieve the critical pressure at the base of the envelope to trigger the outburst (MacDonald 1983), $\sim 10^{-4}-10^{-5} M_{\odot}$ for white dwarf masses from 0.9 to $1.3 M_{\odot}$, in agreement with accreted envelope masses obtained with hydrodynamic models. With the expected constant bolometric luminosity, $10^{4}-10^{5} L_{\odot}$, the nuclear time scale during which the nova was expected to be a bright soft X-ray source was somewhere around tens or hundreds of years. According to these estimations, classical novae would be expected to shine as soft $\mathrm{X}$-ray sources for decades or centuries. The spectrum of this super-soft X-ray emission is expected to be that of a hot white dwarf atmosphere (MacDonald \& Vennes 1991).

The first detection of soft X-rays from a classical nova in outburst came from GQ Mus 1983, when observed with EXOSAT in 1984, 460 days after optical maximum (Ögelman et al. 1984). Other novae were also detected about a hundred days after optical maximum (PW Vul, QU Vul, 
Ögelman et al. 1984), but a major breakthrough was provided with the launch of the Röntgensatellit (ROSAT), which observed a total of 39 recent classical novae, less than 10 years after the outburst. A systematic search for the X-ray emission from those post-outburst classical novae in the ROSAT archival data was performed by Orio et al. (2001). Contrary to what was expected, very few novae were discovered as X-ray emitters shortly after their outburst. In fact only 3 novae showed a soft X-ray spectrum: GQ Mus, V1974 Cyg, and Nova LMC 1995. GQ Mus showed the longest constant bolometric luminosity phase so far observed, its soft X-ray emission lasting for 9 years, still less than predicted. V1974 Cyg, the best monitored soft X-ray nova, turned off only 18 months after the outburst. Nova LMC 1995 was detected by ROSAT as a soft $\mathrm{X}$-ray source three years after outburst, and in a second observation with XMM-Newton in the year 2000, five years after outburst.

X-ray observations give direct insights into the hydrogen burning layer on the white dwarf surface after the outburst, but the presence of the soft X-ray source can also be determined from ultraviolet observations (Shore 2002; Cassatella et al. 2004). The ultraviolet is the key spectral regime for studying the dynamics and opacity of the expanding shell, ionized by the central X-ray source, and determining abundances, velocities, spatial structure, and mass of the ejecta. The ultraviolet emission lines are indicators of the ionization and density structure of the ejecta, while their variations reflect not only the evolution of the expanding shell, but also that of the luminosity and effective temperature of the central ionizing X-ray source. This indirect indicator has been used to determine the turn-off of classical novae from IUE observations (Shore et al. 1996; González-Riestra et al. 1998; Vanlandingham et al. 2001). In all cases, turn-off times were shorter than expected, and also shorter than the 9 years of GQ Mus.

The discrepancy between theory and observations has pointed out that an extra mass loss mechanism should be present shortly after the nova outburst. Some mechanisms have been suggested that involve interaction of the ejecta with the envelope of the secondary and/or thick winds. MacDonald et al. (1985) calculated that a fraction of the envelope could be lost by dynamical friction of the secondary in a common envelope phase following the nova outburst. Kato \& Hachisu (1994) calculated a sequence of optically thick wind solutions that simulated the decay phase of the visual light curve of novae.

Nevertheless, the discrepancy between observations and theory arose from a comparison mainly based on the turn-off times. A model for post-outburst envelopes to which X-ray observations could be compared was lacking. However, a good number of works were devoted to the study of hot white dwarf envelopes. Stellar evolution models following the post AGB phase simulate a similar situation. For Population I stars (with $X_{\mathrm{H}}=0.7$ ), Paczyński (1970) showed that after exhaustion of He in the core, stars of several initial masses (between 3 and $7 M_{\odot}$ ) follow a common track in the HR diagram with constant luminosity. Effective temperature increases along the track, while hydrogen and helium shell burning reduce the envelope mass. Paczyński (1970) was also the first to find that luminosity is directly proportional to the core mass during this phase, which lasts as long as the envelope mass is larger than some critical value. When this critical point is reached, the shell sources die out and the degenerate core starts to cool down.

A number of studies have been devoted to accreting white dwarfs and the development of thermal pulses or flashes on a hydrogen rich envelope. Among them, Iben (1982) and Iben \& Tutukov (1989) found a core mass-luminosity relation for accreting white dwarfs with steady hydrogen burning in the accreted envelope (with $X_{\mathrm{H}}=0.64$ ) similar to Paczyński's. Iben (1982) simulated the evolution of a white dwarf envelope without accretion (or very small values of accretion rate) as a sequence of steady-state models, and found that the constant luminosity phase lasted for decades, in agreement with the nuclear timescales expected for the envelope masses of the postoutburst classical novae predicted by hydrodynamic models. The steady hydrogen-burning solutions were combined with an evolving quasi-static approximation to study the thermal pulses occurring on accreting white dwarfs. Different points of view on the problem can be found in Fujimoto (1982) and Paczyński (1983), who developed simple approximations for the study of the hydrogen-burning envelopes' stability and for the evolution of shell flashes for different accretion rates. Taking the existing numerical models for degenerate stars with hydrogen-burning shells into account, Iben \& Tutukov (1996) pointed out that the core mass-luminosity relation was not unique and that it depended on the thermal history of the star. They compared the models with data from symbiotic stars and cataclysmic variables, exploring the effects of mechanisms not present in the models (such as wind mass loss and mixing mechanisms) in the evolution of accreting white dwarfs. Nevertheless, none of those works have studied envelopes with abundances typical of classical novae. Tuchman \& Truran (1998) studied the composition influence on the core mass-luminosity relation and its implications for the turn-off times of classical novae. They applied their relation to GQ Mus and V1974 Cyg and concluded that the envelope masses required to initiate the thermonuclear runaway were larger than the envelope masses below which stable models for steady hydrogen burning exist. This condition led them to suggest that the remnant envelope masses in the post-outburst stage could be significantly reduced by some kind of dynamical instability.

In this paper, a numerical model for white dwarf envelopes with steady hydrogen burning is presented. The model provides a composition dependent core mass-luminosity relation, and mainly aims at simulating the conditions of the remaining hydrogen-rich material left on the white dwarf after the nova outburst. Photospheric properties were obtained for comparison with X-ray observations, and their evolution is approximated as a sequence of steady-states. Section 2 describes the envelope model, while the main results are presented in Sect. 3 . In Sect. 4, analytical relations between the main global properties of the envelopes are given. The quasi-static evolution is presented in Sect. 5, and a summary and discussion of the results can be found in Sect. 6 .

\section{The white dwarf envelope model}

A grid of white dwarf envelope models with steady hydrogen burning has been computed for five different core masses 
Table 1. Chemical compositions of the white dwarf envelope models in mass fractions. $Z_{\mathrm{s}}$ contains metals (all elements except $\mathrm{H}$ and $\mathrm{He}$ ) in solar fraction. $\delta X_{i}$ indicates the extra mass fraction of element $i$ beyond that included in $Z_{\mathrm{s}}$. The total abundance of metals is indicated by $Z_{\text {total }}$.

\begin{tabular}{ccccccccc}
\hline \hline WD & Mixing & Name & $X$ & $Y$ & $Z_{\mathrm{s}}$ & & & $Z_{\text {total }}$ \\
\hline & & & & & & $\delta X_{\mathrm{O}}$ & $\delta X_{\mathrm{Ne}}$ & \\
\hline $\mathrm{ONe}$ & $75 \%$ & ONe75 & 0.18 & 0.08 & 0.12 & 0.38 & 0.24 & 0.74 \\
$\mathrm{ONe}$ & $50 \%$ & ONe50 & 0.35 & 0.15 & 0.09 & 0.25 & 0.16 & 0.5 \\
$\mathrm{ONe}$ & $25 \%$ & ONe25 & 0.53 & 0.2 & 0.06 & 0.13 & 0.08 & 0.27 \\
\hline & & & & & & $\delta X_{\mathrm{C}}$ & $\delta X_{\mathrm{O}}$ & \\
\hline $\mathrm{CO}$ & $50 \%$ & $\mathrm{CO} 50$ & 0.35 & 0.14 & 0.02 & 0.245 & 0.245 & 0.51 \\
\hline
\end{tabular}

(from 0.9 to $1.3 M_{\odot}$ ) and four different chemical compositions, all of them corresponding to realistic envelopes accreted onto white dwarfs in novae. Compositions are taken from José \& Hernanz (1998), who developed hydrodynamic simulations of nova explosions with detailed computation of the nucleosynthesis, and obtained results for the composition of the ejecta compatible with observed abundances in many cases. As already found from the first hydrodynamic models of nova outbursts, their models showed that the material accreted from the secondary with solar abundances needs to be enhanced with CNO nuclei by some mixing mechanism between the envelope and the degenerate core. The compositions considered here correspond to $\mathrm{CO}$ white dwarf envelopes with $50 \%$ mixing of the solar accreted matter with the core material, and to $\mathrm{ONe}$ white dwarf envelopes with $25 \%, 50 \%$, and $75 \%$ mixing. For the calculation of the accreted envelope composition resulting from the mixing, José \& Hernanz (1998) took the composition of the degenerate cores from Ritossa et al. (1996) for the ONe white dwarfs, and assumed $49.5 \%$ oxygen, $49.5 \%$ carbon, and $1 \%$ neon- 22 in the $\mathrm{CO}$ cases. Table 1 shows the details of the chemical compositions.

\subsection{Numerical method and boundary conditions}

The numerical code integrates the equations of stellar equilibrium from the photosphere to the base of the envelope. The envelope is tested at each step for convective instability according to the Schwarzschild criterion. When convection appears, the temperature gradient is solved using the standard mixinglength theory of convection (Böhm-Vitense 1958). Radiative opacities were calculated for each composition with the online version of the OPAL code from the Lawrence Livermore National Laboratory (Iglesias \& Rogers 1996), and the energy generation rates for hydrogen burning are the analytical approximations from Reeves (1965) and Paczyński (1983). The equation of state is that of an ideal gas with radiation pressure. It was checked that for the gas conditions in the present models, no significant degeneracy at the base of the envelope or partial ionization effects at the outermost layers needed to be taken into account and that the energy production due to helium burning is negligible.

The stellar equilibrium equations were integrated inwards using an embedded (adaptative stepsize controlled) RungeKutta method with Cash-Karp parameters (Cash \& Karp 1990; Press et al. 1992). Integration started at the photosphere with a fixed value for the total luminosity and trial values for the total mass of the envelope and the photospheric radius, and proceeded inwards until the luminosity reached a sufficiently small value, which was considered to indicate the base of the hydrogen burning layer. As seen later, for all white dwarf masses and compositions there is a plateau of constant luminosity where it experiences little change for a wide range of effective temperatures. On this plateau, the total luminosity does not determine a unique solution anymore, and the photospheric radius was fixed instead.

The boundary conditions at the photosphere were obtained from a grey atmosphere model of the same composition as the envelope, which provided the value of the atmospheric pressure at the photosphere for the fixed value of the luminosity and the trial values of the envelope mass and photospheric radius. The atmosphere was tested for convective instability during integration, which never appeared before reaching the photosphere. At the base of the envelope, the envelope mass was required to be smaller than $10^{-13} M_{\odot}$, while the radius, $R_{\text {base }}$, was compared to the core radius, $R_{\mathrm{c}}$, by requiring factor $\left|\frac{R_{\text {base }}-R_{\mathrm{c}}}{R_{\mathrm{c}}}\right|$ to be smaller than $10^{-5}$. If these conditions were not fullfiled, the integration restarted from the photosphere with new trial values for the envelope mass and photospheric radius. The core radius was taken from the Hamada \& Salpeter (1961) core massradius relation for zero temperature white dwarfs. We checked that a slight increase in the core radius does not affect our main results; this radius increase is only expected if degeneracy is lifted in the outer core shells as a consequence of heating, which is not probable since the timescale for heating propagation towards the core is quite large (Henyey \& L'Ecuyer 1969).

\section{Results}

The luminosity versus effective temperature for all models, i.e. their position in the HR diagram, is shown in left panel of Fig. 1. For each composition and white dwarf mass, the envelope solutions have a maximum effective temperature that divides the series of models into two branches: a branch of high, quasi-constant luminosity, where the maximum value for the luminosity is reached; and a branch of low luminosity and constant photospheric radius. The models of the high luminosity branch have in all cases a convective region below the photosphere, while the energy transfer in most of the envelopes of the low luminosity branch is radiative in the whole structure. The right panel in Fig. 1 shows the envelope mass as a 

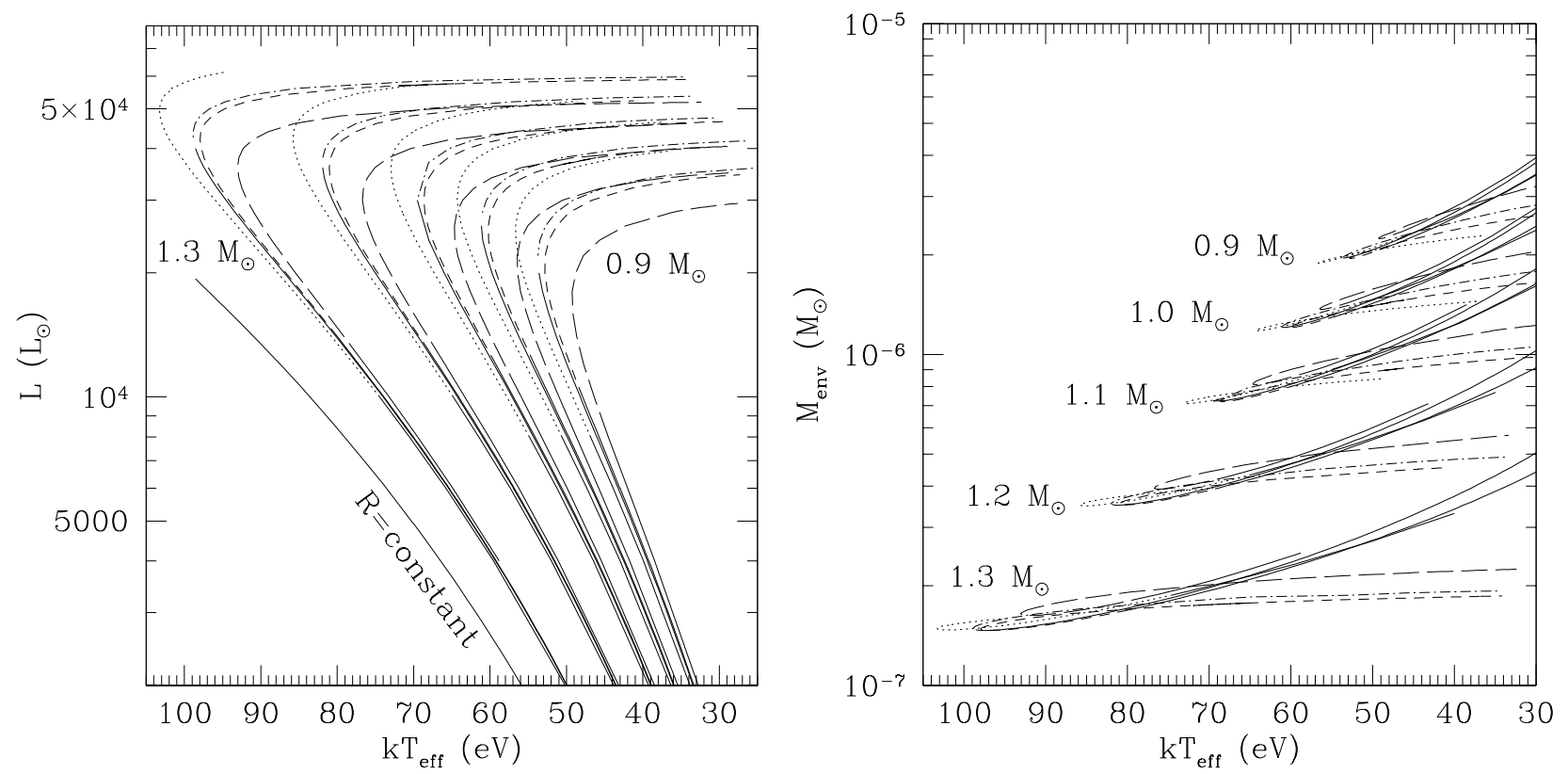

Fig. 1. Total luminosity (left panel) and envelope mass (right panel) versus effective temperature for our white dwarf envelope models: ONe75 (dotted line), ONe50 (short dash), ONe25 (long dash), and CO50 (short dash-dot). Effective temperature is given in eV, $k T_{\text {eff }}(\mathrm{eV})=8.617 \times 10^{-5} T_{\text {eff }}(\mathrm{K})$ Solid lines indicate envelopes without convective regions. Five series of models are plotted for each chemical composition, corresponding to total masses $0.9,1.0,1.1,1.2$, and $1.3 M_{\odot}$. A line indicating the luminosity-effective temperature relation for constant photospheric radius is over-plotted in left panel for comparison.

function of effective temperature. For every white dwarf mass there is a minimum value for the envelope mass, which occurs in the vicinity of the maximum effective temperature. The envelope mass along the high luminosity branch is almost constant, decreasing only slowly for increasing effective temperatures, while the decrease in the envelope mass for increasing effective temperatures along the low luminosity branch is steeper. A summary of the main properties of all model envelopes is found in Table 2 .

\subsection{Stability}

A stability analysis of the shell source indicates that the burning shell is only thermally stable for the solutions along the high luminosity branch. The instability of the low luminosity branch can also be understood in a qualitative way if the evolution of the envelope is approximated as a succession of steady states with decreasing envelope masses. Figure 2 shows the temperature at the base of the envelope for CO50 models. The high luminosity branch in Fig. 1 corresponds to envelopes with high base temperatures in Fig. 2, while envelopes with low base temperatures correspond to the low luminosity branch. From the left panel in Fig. 1 it is clear that, along any of the two branches, the envelope mass decreases towards higher effective temperatures. If the evolution of an envelope is approximated as a succession of steady states, it will proceed in any case towards decreasing envelope masses, i.e., increasing the effective temperature. Hydrogen burning will continue until the minimum envelope mass and maximum effective temperature are reached; evolution cannot proceed further with stationary hydrogen burning, since no equilibrium configuration exists for a lighter envelope.

Along the high luminosity branch, an envelope evolves at quasi-constant luminosity, increasing the effective temperature as a consequence of the decrease of the photospheric radius, which sinks the photosphere deeper into the envelope. Nevertheless, the envelope as a whole is slowly cooling down and energy production decreases with time, with both the luminosity and the temperature at the base of the envelope slowly decreasing (Figs. 1 - left panel - and 2). But along the low luminosity branch (the low bottom temperature branch in Fig. 2) for decreasing envelope masses, the temperature at the base and the luminosity of the envelope would increase. This would require an extra source of energy and indicates that evolution cannot proceed along this branch.

The stable, high luminosity branch is limited at its low effective temperature end by the Eddington luminosity. For the low temperature models along this branch, the radiative luminosity closely approaches the Eddington limit as effective temperature decreases, mainly because there's a photospheric opacity increase leading to an Eddington luminosity decrease. Therefore, envelopes in hydrostatic equilibrium do not exist for lower effective temperatures, and thus larger envelope masses and photospheric radii, than those shown in Fig. 1.

\subsection{Internal structure}

Figure 3 shows the internal structure of some of the CO50 and ONe50 envelope models of the high luminosity branch, all of them with the same effective temperature $\left(\sim 4.6 \times 10^{5} \mathrm{~K} \sim 40 \mathrm{eV}\right)$. The temperature and luminosity 
Table 2. Main properties of the envelope models.

\begin{tabular}{cccccccc}
\hline \hline Model & $\begin{array}{c}M_{\mathrm{c}} \\
\left(M_{\odot}\right)\end{array}$ & $\begin{array}{c}L^{\text {plateau }} \\
\left(10^{4} L_{\odot}\right)\end{array}$ & $\begin{array}{c}k T_{\text {eff }}^{\max } \\
(\mathrm{eV})\end{array}$ & $\begin{array}{c}T_{\text {eff }}^{\max } \\
\left(10^{5} \mathrm{~K}\right)\end{array}$ & $\begin{array}{c}M_{\mathrm{env}}^{\max } \\
\left(10^{-6} M_{\odot}\right)\end{array}$ & $\begin{array}{c}M_{\text {env }}^{\min } \\
\left(10^{-6} M_{\odot}\right)\end{array}$ & $\begin{array}{c}\Delta t_{10 \mathrm{eV}}^{(a)} \\
(\text { days })\end{array}$ \\
\hline ONe75 & 0.9 & 3.9 & 57 & 6.6 & 2.3 & 1.9 & 47 \\
$(X=0.18)$ & 1.0 & 4.4 & 64 & 7.4 & 1.4 & 1.2 & 24 \\
& 1.1 & 5.2 & 73 & 8.5 & 0.84 & 0.71 & 12 \\
& 1.2 & 5.7 & 86 & 9.9 & 0.40 & 0.35 & 4.6 \\
& 1.3 & 6.2 & 103 & 11.9 & 0.16 & 0.15 & 1.1 \\
\hline & 0.9 & 3.5 & 53 & 6.1 & 2.7 & 1.5 & 160 \\
ONe50 & 1.0 & 4.1 & 60 & 7.0 & 1.7 & 1.2 & 78 \\
& 1.1 & 4.7 & 69 & 8.0 & 0.99 & 0.72 & 37 \\
& 1.2 & 5.2 & 81 & 9.4 & 0.45 & 0.35 & 14 \\
ONe25 & 1.3 & 5.6 & 98 & 11.4 & 0.19 & 0.15 & 4.9 \\
\hline$X=0.53)$ & 1.0 & 3.5 & 56 & 6.5 & 2.1 & 1.4 & 210 \\
& 1.1 & 4.0 & 64 & 7.5 & 1.20 & 0.81 & 98 \\
& 1.2 & 4.6 & 77 & 8.9 & 0.60 & 0.39 & 36 \\
& 1.3 & 5.2 & 93 & 10.8 & 0.22 & 0.16 & 12 \\
\hline & 0.9 & 3.6 & 54 & 6.2 & 2.9 & 1.9 & 230 \\
$(X=0.35$ & 1.0 & 4.2 & 61 & 7.1 & 1.8 & 1.2 & 90 \\
& 1.1 & 4.7 & 70 & 8.1 & 1.0 & 0.72 & 48 \\
& 1.2 & 5.4 & 82 & 9.5 & 0.49 & 0.35 & 19 \\
& 1.3 & 6.0 & 99 & 14.7 & 0.19 & 0.15 & 6 \\
\hline
\end{tabular}

${ }^{a}$ Time needed for the envelope to evolve from $k T_{\text {eff }} \simeq k T_{\text {eff }}^{\max }-10 \mathrm{eV}$ to $k T_{\text {eff }}^{\max }$.

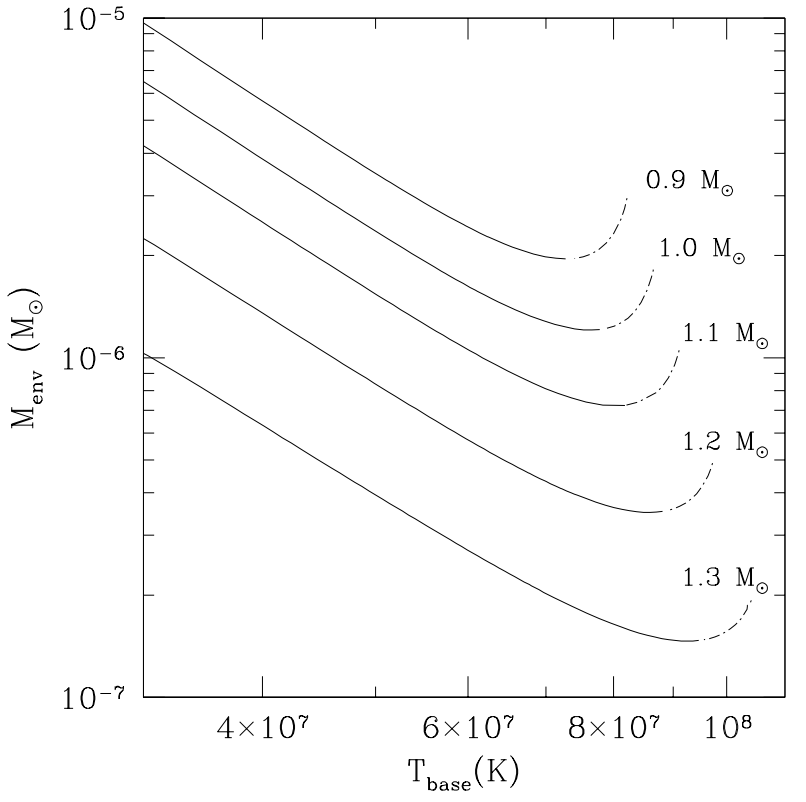

Fig. 2. Envelope mass versus base temperature for CO50 models. Solid lines indicate envelopes without convective regions.

profiles show that only the deepest layers have high enough temperatures to contribute to energy production. Luminosity stays constant in most of the envelope. The highest temperatures at the base are needed for the most luminous envelopes, and in a similar way the most massive envelopes produce the highest pressures at the bottom.
As mentioned above, envelopes on the high luminosity branch have a convective region just below the photosphere, where opacity reaches its highest values. Since convection transfers energy outwards more efficiently than radiation, the temperature gradient is flatter in the convective regions, as are the pressure and density gradients, which contributes to a larger photospheric radius and luminosity. It can be observed that for the largest luminosities and most massive white dwarfs, the density gradient in the convective region is close to zero. For high luminosities and gravities, inversions of the density gradient have been frequently found in convective layers of white dwarf envelopes when using the mixing length theory (Fontaine \& van Horn 1976; Henyey et al. 1965; Latour 1970; Mihalas 1965). However, although the density gradient is very flat in some of the present models, such inversions never appear. The convective region is more extended for increasing white dwarf masses. As a consequence, temperature, pressure, and density gradients in the inner radiative layers are steeper for massive white dwarfs, causing the energy production layer to be thinner in these cases, which is clearly visible in the luminosity profiles. In a similar way, the envelope mass increases only in the deepest layers in massive white dwarfs, since most of the envelope has low density values.

The effects of the different chemical compositions on the envelopes' structure are shown in Fig. 4, where profiles for five models with different abundances, but the same core mass and total luminosity, are plotted. The strongest difference in behaviour corresponds to the model that is richest in hydrogen, ONe25. In this case, the higher hydrogen content causes 

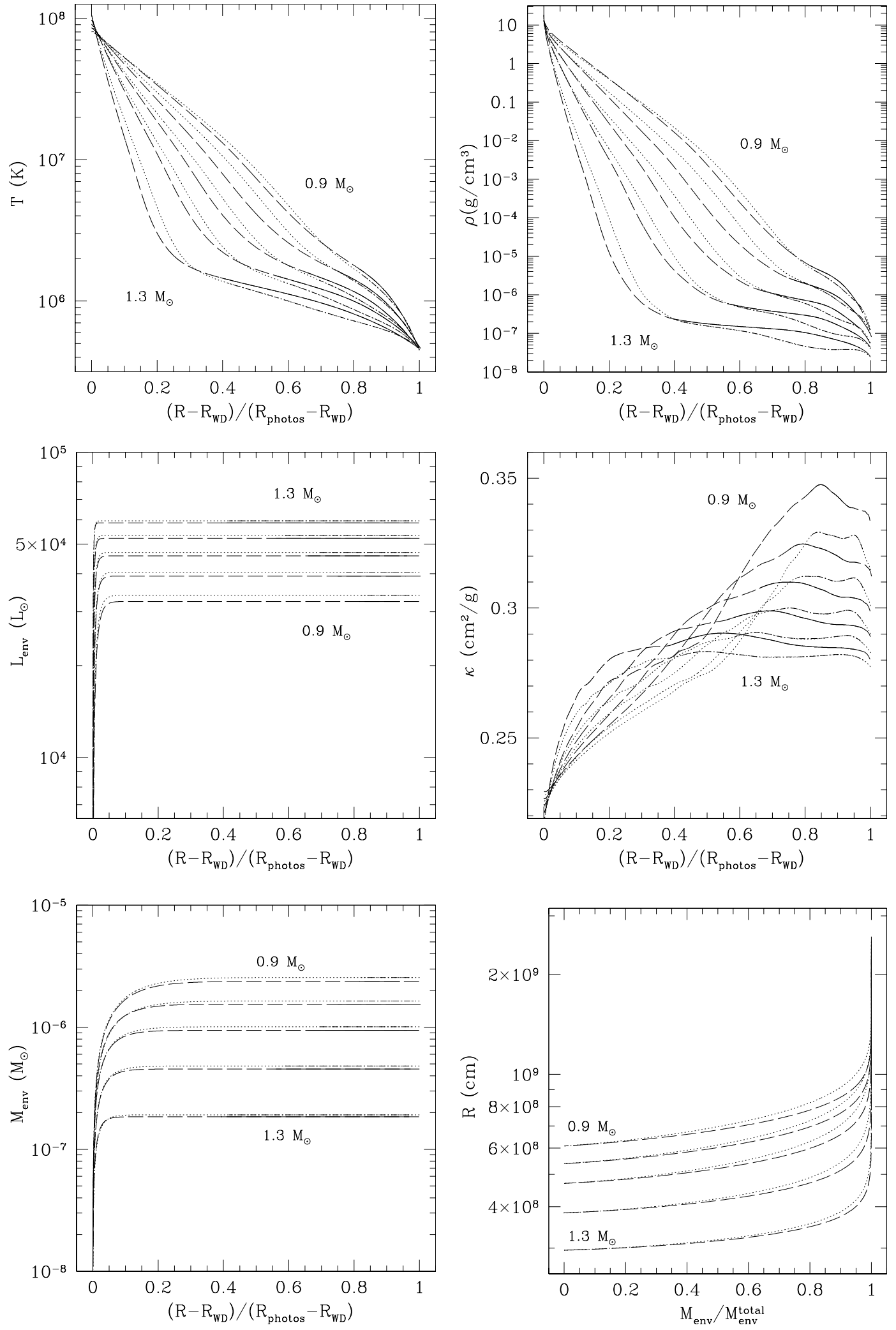

Fig. 3. Internal structure of ONe50 (dashed line) and CO50 (dotted line) envelopes with core masses $0.9,1.0,1.1,1.2$, and $1.3 M_{\odot}$, all of them with a similar effective temperature, $\sim 4.6 \times 10^{5} \mathrm{~K}(\sim 40 \mathrm{eV})$. Photospheric radii are, for ONe50 models, between $1.9 \times 10^{9} \mathrm{~cm}$ for $0.9 M_{\odot}$ and $2.6 \times 10^{9} \mathrm{~cm}$ for $1.3 M_{\odot}$; and for CO50 models between $2.1 \times 10^{9} \mathrm{~cm}$ for $0.9 M_{\odot}$ and $2.5 \times 10^{9} \mathrm{~cm}$ for $1.3 M_{\odot}$. Solid (ONe50) and dash-dotted (CO50) lines indicate convective zones. 

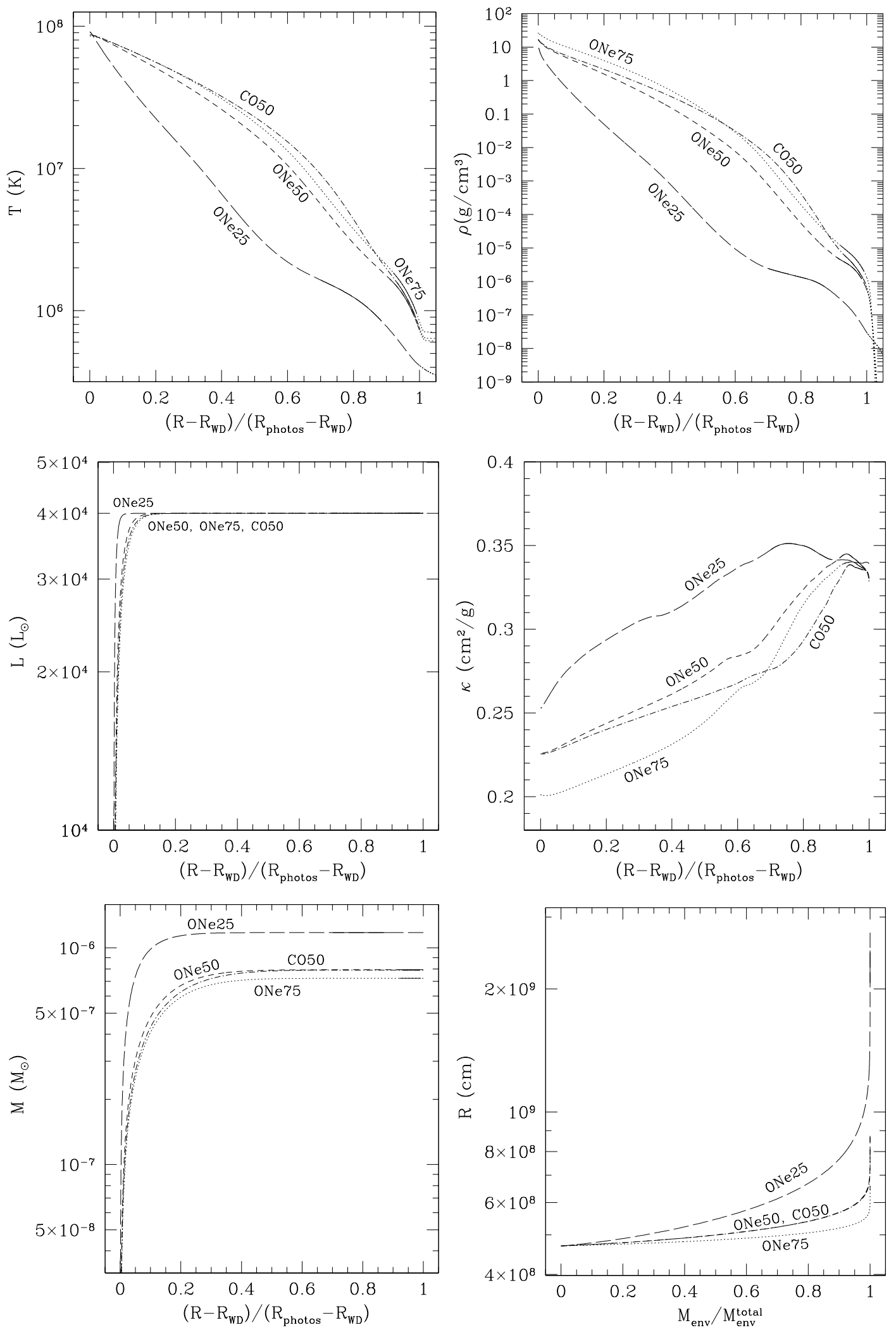

Fig. 4. Internal structure of four envelope models with the same core mass $\left(1.1 M_{\odot}\right)$ and similar luminosity $\left(4 \times 10^{4} L_{\odot}\right)$, but with different chemical compositions: CO50 with photospheric radius $8.1 \times 10^{8} \mathrm{~cm}$ (dash dotted line), ONe25 with $2.7 \times 10^{9} \mathrm{~cm}$ (long-dashed line), ONe50 with $8.7 \times 10^{8} \mathrm{~cm}$ (short-dashed line), and ONe75 with $6.6 \times 10^{8} \mathrm{~cm}$ (dotted line). A solid line indicates convection regions, and the atmosphere is plotted with dotted line for all cases.

the opacities to be larger than in the envelopes that are poorer in hydrogen, causing the convective region to be much more extended. As a consequence, profiles in the radiative regions are steeper, especially in the deepest layers. 
Different chemical compositions and white dwarf mass combinations give envelopes with the same observable properties (luminosity and effective temperature), as can be seen in Fig. 1. Figure 5 plots the internal structure of three $\mathrm{ONe}$ envelopes with the same effective temperature and luminosity $\left(\sim 5.2 \times 10^{5} \mathrm{~K} \sim 45 \mathrm{eV}, \sim 5.2 \times 10^{4} L_{\odot}\right)$, and thus the same photospheric radius $\left(2 \times 10^{9} \mathrm{~cm}\right)$, but different white dwarf masses and compositions $\left(\mathrm{ONe} 25\right.$ with $1.3 M_{\odot}$, ONe50 with $1.2 M_{\odot}$, and ONe75 with $1.1 M_{\odot}$ ). In spite of the differences in abundances, the profiles are very similar, resulting in equal photospheric properties. The convection region is more extended for the model richest in hydrogen, ONe25, which is in general cooler, except for the innermost layers. For comparison, an ONe50 model of the unstable, low luminosity branch and with the same effective temperature is also plotted.

There are small differences in the general properties of the $\mathrm{CO} 50$ and the ONe50 envelope models, despite having the same hydrogen mass fraction. From Fig. 1 it is clear that, for the same effective temperature, the CO50 models have slightly higher luminosities and larger envelope masses. Likewise, for the same luminosity, $\mathrm{CO} 50$ envelope models have higher effective temperatures and smaller photospheric radii. This is evident in Fig. 3, where CO50 and ONe50 models are plotted for several masses, all of them with the same effective temperature, for which distributions of temperature and density (in fraction of total envelope radius) are steeper for ONe50 models. This is directly related to larger opacities for ONe50 than for CO50 compositions in the temperature range of the radiative region of the envelopes, with $T>10^{6} \mathrm{~K}$, which cause the temperature distribution to change more rapidly with radius. Since temperature decreases more rapidly in the inner regions of ONe50 models than for CO50, the energy production layer is thinner and the total luminosity of the envelope is smaller. Analogously, the density profile in the inner regions is also steeper for ONe50 models, causing the envelope mass to increase only in a narrower region than in $\mathrm{CO} 50$ models and the total envelope mass to be smaller.

To check that the differences between $\mathrm{CO} 50$ and ONe50 models are due to the differences in the opacities, some envelope models were reintegrated with opacities artificially altered by a certain factor. We found that, as in the case of the CO50-ONe50 comparison, envelopes with higher opacities have smaller luminosities and envelope masses. A global increase (decrease) in the opacity in the envelope by $10 \%$ causes a decrease (increase) in the envelope mass of $4 \%$ and a $10 \%$ decrease (increase) in the luminosity.

\section{Analytical relations between main envelope properties}

\subsection{Core mass-luminosity relation}

From Fig. 1 it is clear that luminosity is almost constant along the stable, high-luminosity branch and that its value increases with the core mass. In the left panel of Fig. 6, plateau luminosities are plotted against core mass for all models. The resulting core mass-luminosity relation for our ONe models can be approximated by

$L_{\mathrm{ONe}}^{\text {plateau }}\left(L_{\odot}\right) \simeq 5.95 \times 10^{4}\left(\frac{M_{\mathrm{c}}}{M_{\odot}}-0.536 X_{\mathrm{H}}-0.14\right)$

and for the CO50 models by

$L_{\text {CO50 }}^{\text {plateau }}\left(L_{\odot}\right) \simeq 5.95 \times 10^{4}\left(\frac{M_{\mathrm{c}}}{M_{\odot}}-0.3\right)$.

The plateau luminosity increases with the white dwarf mass and for increasing mixing factors of the accreted solar matter with the core material, i.e., for decreasing hydrogen abundances. Envelopes with a lower hydrogen mass fraction need higher temperatures to reach an equilibrium configuration, and therefore have larger luminosities. The plateau luminosity versus the hydrogen mass fraction is shown in the right panel of Fig. 6 for all models. Relation (1) is over-plotted for each core mass. While ONe models follow (1) (except for the most massive white dwarf), CO50 models follow a parallel relation but are slightly more luminous than ONe50 (between a 3\% and a $7 \%$ more luminous), which can also be seen in Fig. 1.

The linear relation between the luminosity of a shellburning star on a degenerate core and its core mass has been known for a long time. An analytical justification for this linear dependence was obtained by Kippenhahn (1981; see also Kippenhahn \& Weigert 1990). He showed that the luminosity was directly proportional to the core mass and independent of its radius for $\beta \ll 1$, where $\beta=\frac{P_{\text {gas }}}{P_{\text {total }}}$. With this analytical work, Kippenhahn (1981) confirmed results found with numerical evolutionary calculations by Paczyński (1970), who showed that the luminosity of post-AGB stars with a doubleburning shell $(\mathrm{H}, \mathrm{He})$ on top of a mostly degenerate $\mathrm{CO}$ core could be expressed as $L\left(L_{\odot}\right)=a\left(M_{\mathrm{c}} / M_{\odot}-b\right)$, with $a=59250$ and $b=0.522$. Later works found similar results, with small changes in the parameters. Among them, Iben (1982) found that in the case of steady state $a=59500$ and $b=0.5$. For the hydrogen mass fraction used by Iben $(1982)\left(X_{\mathrm{H}}=0.64\right)$, the $0.536 X_{\mathrm{H}}+0.14$ factor in Eq. (1), 0.48 , is very similar to the value found in that work, 0.5 .

The increase in the luminosity with decreasing hydrogen mass fraction was also found in analytical studies by Tuchman et al. (1983) and with numerical calculations of steady hydrogen burning on white dwarfs by Tuchman \& Truran (1998). They studied the dependence of luminosity on the hydrogen mass fraction for a fixed metallicity $(Z=0.25)$ and for a constant $\mathrm{He} / \mathrm{H}$ ratio (assumed to be solar, $\approx 0.1$ ), and summarized their dependences by the relation $L\left(L_{\odot}\right)=5.2 \times 10^{4}\left[M_{\mathrm{c}} / M_{\odot}-\right.$ $0.205-0.5(X-Z)]$. According to the authors, this relation also holds for other metallicities. More recently, Marigo (2000) used an homology formalism to justify the linear core mass luminosity relation and to study the dependence of the luminosity on the chemical composition for giant stars with core masses between 0.5 and $0.7 M_{\odot}$.

\subsection{Other relations}

The maximum effective temperature also increases with decreasing hydrogen mass fractions and with increasing core 

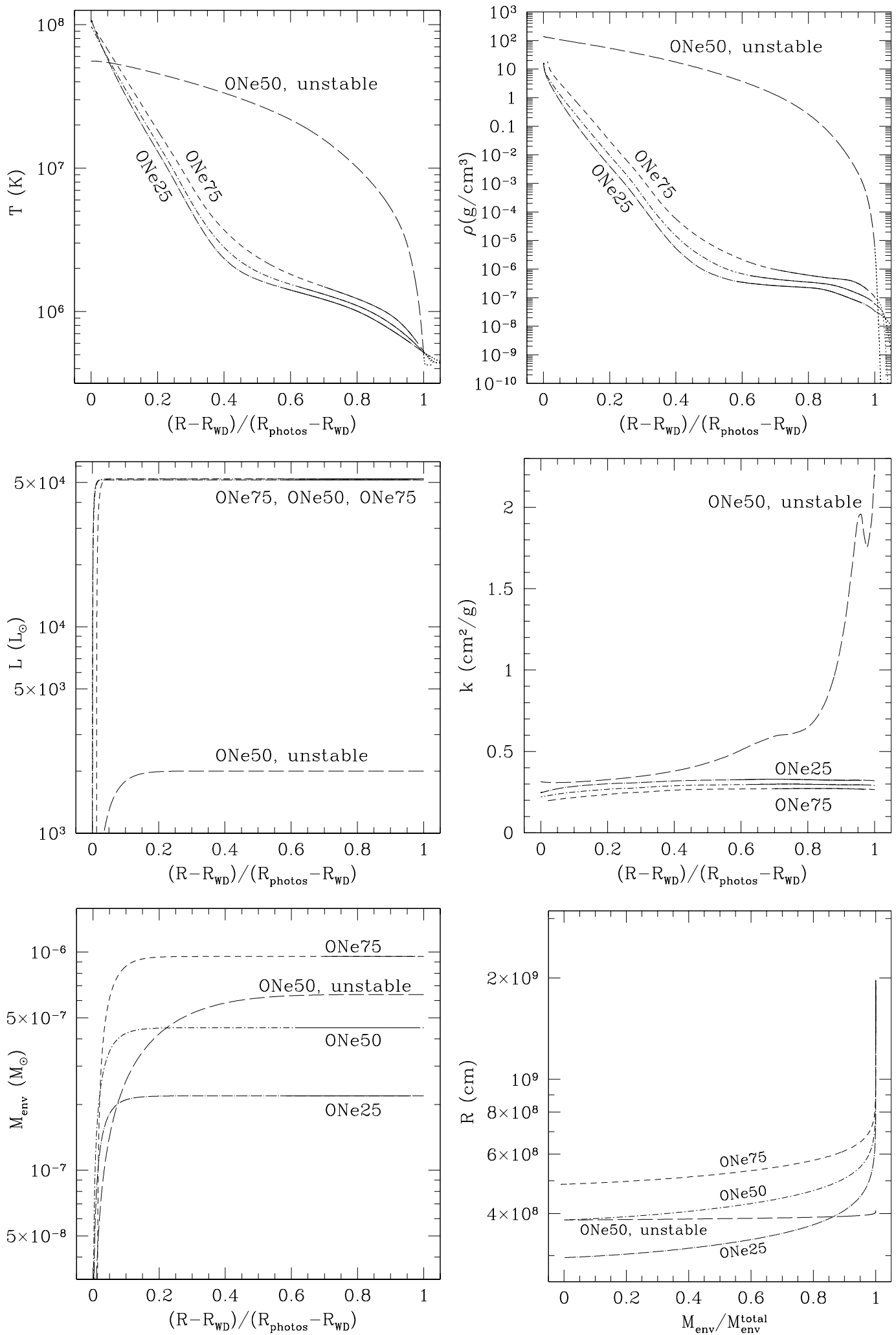

Fig. 5. Internal structure of some ONe white dwarf envelope models with different white dwarf masses and composition and with the same luminosity, $\sim 5.2 \times 10^{4} L_{\odot}$, and effective temperature, $\sim 5.2 \times 10^{5} \mathrm{~K}(\sim 45 \mathrm{eV})$. Three models belong to the high luminosity branch, all three with the same total luminosity: ONe25 with $M_{\mathrm{c}}=1.3 M_{\odot}$ (long dash-dotted line), ONe50 with $M_{\mathrm{c}}=1.2 M_{\odot}$ (short dash-dotted line), and ONe75 with $M_{\mathrm{c}}=1.1 M_{\odot}$ (short dashed line). All of them have photospheric radius, $2 \times 10^{9} \mathrm{~cm}$. A fourth model from the low luminosity branch, but with the same effective temperature, is also plotted (ONe50 with $M_{\mathrm{c}}=1.2 M_{\odot}, L=2 \times 10^{3} L_{\odot}$ and $R=4 \times 10^{8} \mathrm{~cm}$, long-dashed line). Solid lines indicate convective zones, and atmospheres are plotted with dotted lines. 

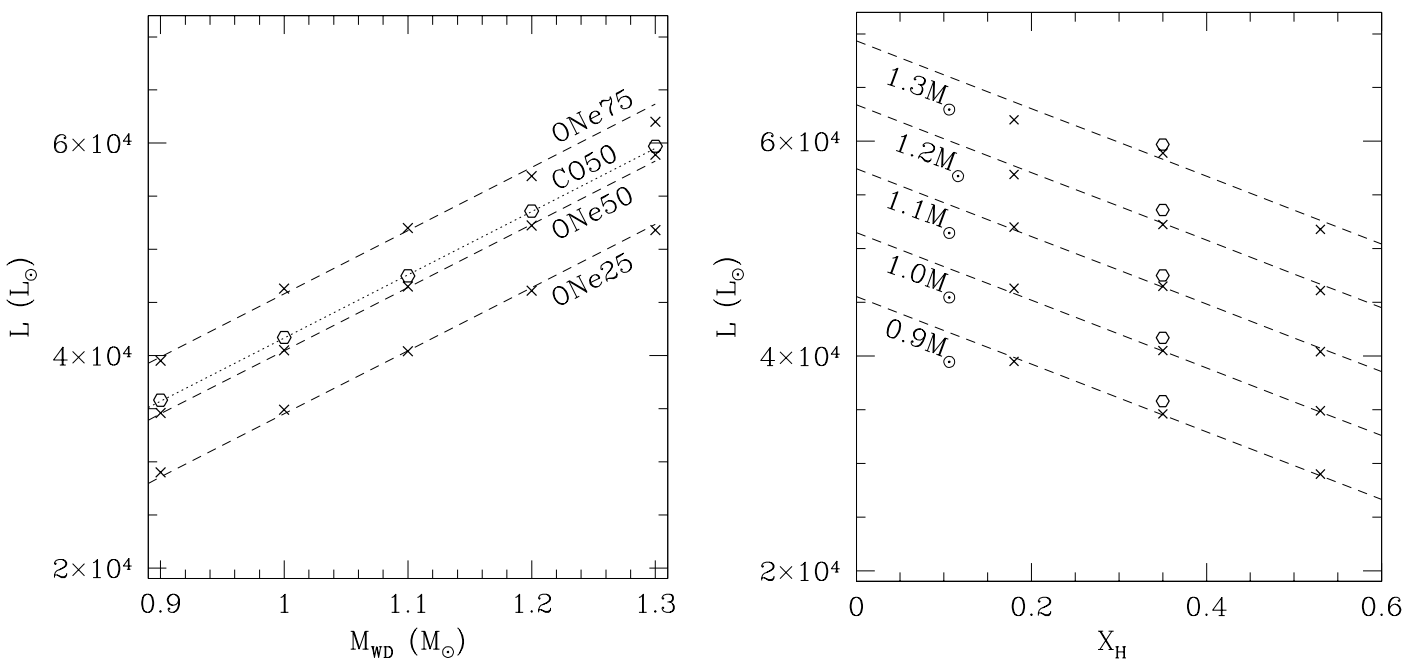

Fig. 6. Plateau luminosity versus core mass (left) and hydrogen mass fraction (right). Crosses indicate ONe envelope models results, while open dots correspond to CO50 models. Dashed lines correspond to Eq. (1) for ONe models, and the dotted line in the left panel to Eq. (2) for CO50 models.

masses (see Fig. 7), but in this case, no clear relation has been found. As shown in the figure, the maximum effective temperature does not follow any unique linear relation or power law with the core mass. Its behaviour can only be approximated by a power law, $k T_{\text {eff }}(\mathrm{eV}) \propto M_{\mathrm{c}}^{9 / 10}$ for core masses larger than $1.1 M_{\odot}$ and $k T_{\text {eff }}(\mathrm{eV}) \propto M_{\mathrm{c}}^{5 / 9}$ for less massive white dwarfs.

As in the case of luminosity, the envelope mass on the luminosity plateau depends on the core mass and on the hydrogen mass fraction (see Fig. 1, right panel). Iben (1982) found that the relationship between the envelope mass and the white dwarf mass on the luminosity plateau was $\log M_{\mathrm{env}}^{\mathrm{Iben} 82}\left(M_{\odot}\right) \simeq$ $-4.38-4.96\left(M_{\mathrm{c}} / M_{\odot}-1\right)$. Since the envelope mass decreases along the luminosity plateau for increasing effective temperatures (see Fig. 1) a clear definition of the envelope mass can not be established. In Fig. 8 (left panel), the maximum envelope mass for our stable models is plotted as a function of the core mass. It is clear that for our models, the $\log M_{\mathrm{env}}^{\max }-M_{\mathrm{c}}$ relation is not linear. A general relation between the maximum envelope mass, the core mass, and the hydrogen mass fraction can be found for our envelopes:

$\log M_{\mathrm{env}}^{\max }\left(M_{\odot}\right) \simeq 0.42 X_{\mathrm{H}}-\left(\frac{M_{\mathrm{c}}}{M_{\odot}}-0.13\right)^{3}-5.26$

which is indicated in Fig. 8.

The minimum envelope mass does not have a strong dependence on the hydrogen mass fraction (see the right panel of Fig. 9), and can be approximated by the relation

$\log M_{\mathrm{env}}^{\min }\left(M_{\odot}\right) \simeq 0.13 X_{\mathrm{H}}-\left(\frac{M_{\mathrm{c}}}{M_{\odot}}-0.13\right)^{3}-5.26$.

\section{Quasi-static temporal evolution}

An estimation of the evolution of a steady hydrogen-burning white dwarf envelope on the plateau of quasi-constant luminosity can be approximated by a series of stationary envelope models with decreasing envelope masses. The layers of the envelopes whose hydrogen has been processed are assimilated to the core and do not take part in the integration of the next envelope model (the increment in the core mass is negligible). The time $\Delta t$ needed for the envelope to change its mass $\Delta M_{\text {env }}$ only due to hydrogen burning can be estimated as

$\Delta t=\epsilon \frac{\Delta M_{\mathrm{env}} X_{\mathrm{H}}}{L}$

where $X_{\mathrm{H}}$ is the hydrogen mass fraction, $L$ the mean luminosity in this period (which varies little in the constant bolometric luminosity phase), and $\epsilon$ the energy released per gram of hydrogen processed $\left(\epsilon=5.98 \times 10^{18} \mathrm{erg} \mathrm{g}^{-1}\right)$. In Figs. 10 and 11, time intervals needed for the envelope to evolve between two adjacent marked points are indicated in days. Evolution proceeds more slowly for smaller white dwarfs with lower luminosities. Time intervals are also longer for envelopes with increasing hydrogen abundance, which have smaller luminosities.

The times found in this work are much shorter than the evolutionary times usually found in the literature for stationary hydrogen-burning on white dwarfs, including for instance the times found by Iben (1982). This difference can be explained by the smaller hydrogen fraction in the envelope: the equilibrium configuration is hotter and brighter for hydrogen poorer envelopes, and envelopes masses are smaller. To keep this higher luminosity, hydrogen is consumed faster; in addition, for the same amount of hydrogen consumed, a larger fraction of the envelope is reduced. As envelope masses are smaller and are reduced at a faster rate, the total timescale to consume the envelope is shorter.

These timescales can be compared with the nuclear-burning lifetime for classical novae hydrostatic remnants found in the literature. In Starrfield (1989), this lifetime was estimated as

$\tau_{\text {nuc }}=400\left(\frac{M_{\mathrm{H}}}{10^{-4} M_{\odot}}\right)\left(\frac{L}{2 \times 10^{4} L_{\odot}}\right)^{-1} \mathrm{yr}$

where $M_{\mathrm{H}}$ is the hydrogen mass and $L$ the total luminosity. A similar expression, only a factor $\frac{5}{4}$ larger, was given by MacDonald et al. (1985). In both cases, the nuclear lifetime 

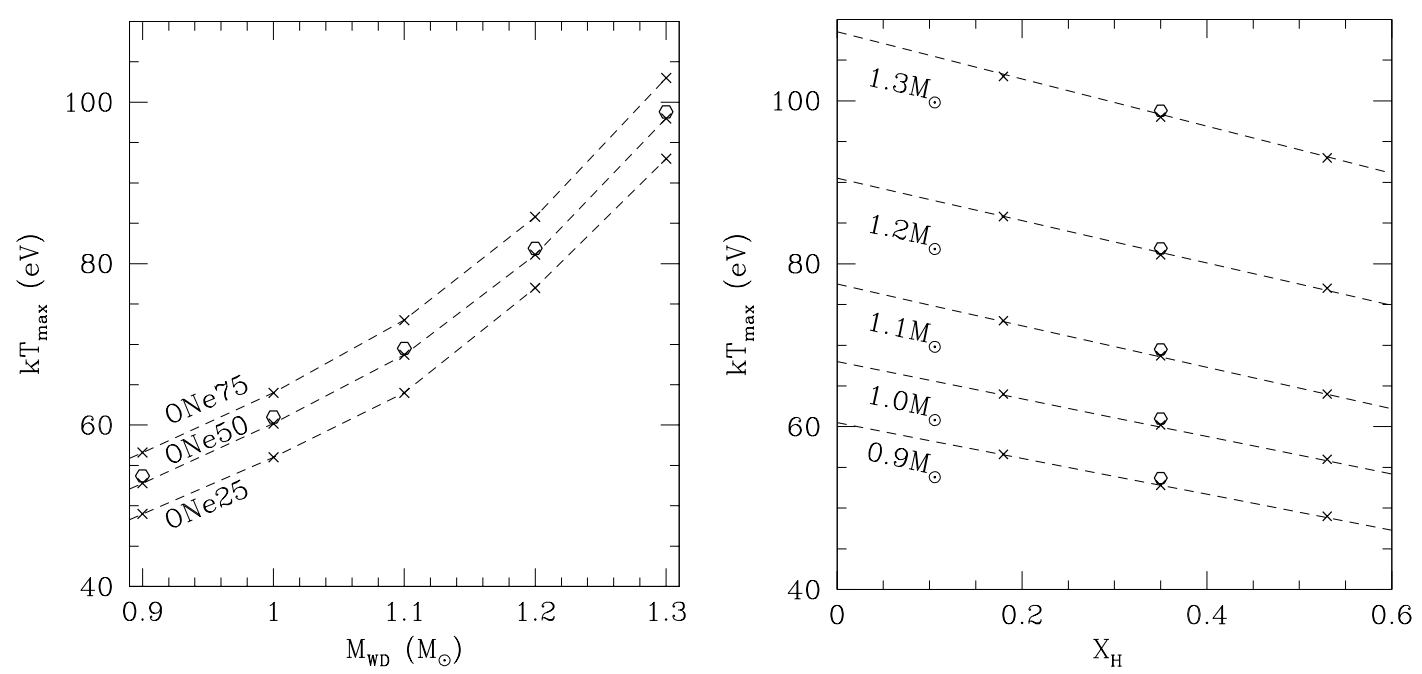

Fig. 7. Maximum effective temperature versus core mass (left) and hydrogen mass fraction (right) for the same models shown in Fig. 6. Lines joining points are for orientation purposes.
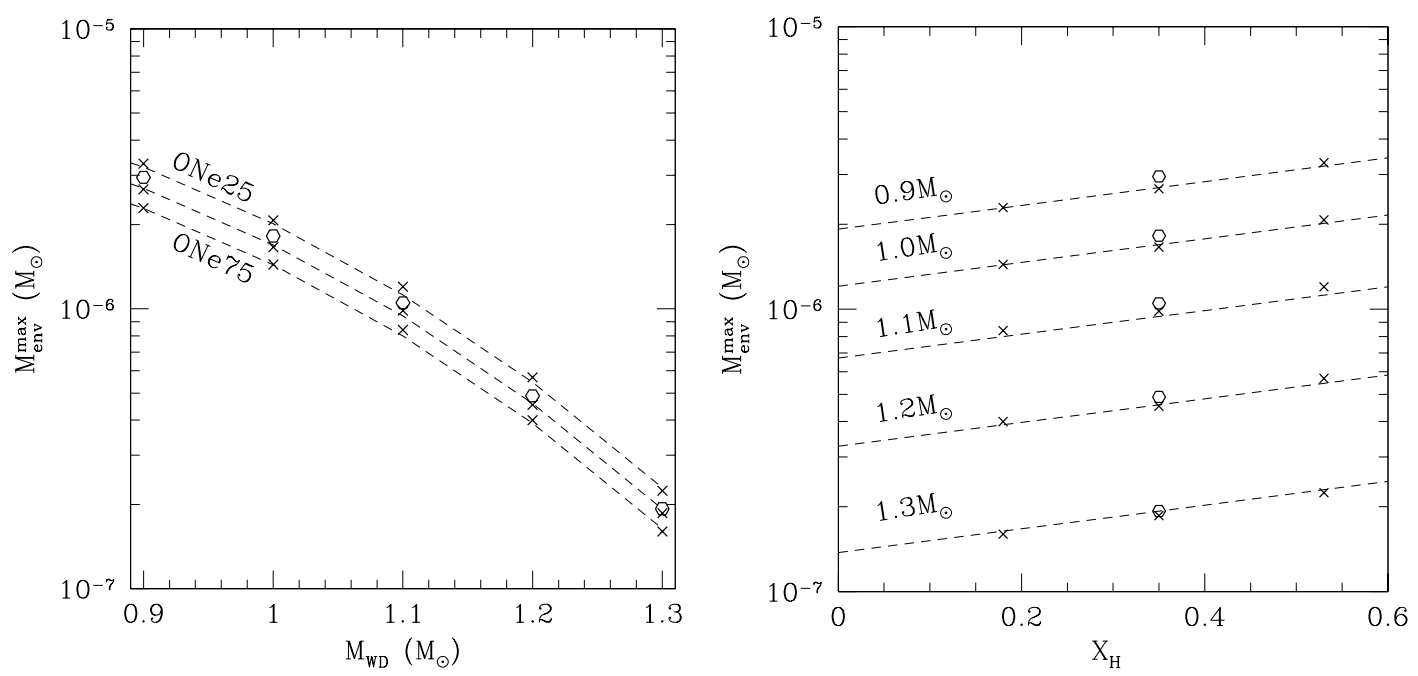

Fig. 8. Maximum envelope mass versus core mass (left) and hydrogen mass fraction (right) for the same models shown in Fig. 6. Equation (3) is shown in dashed lines.
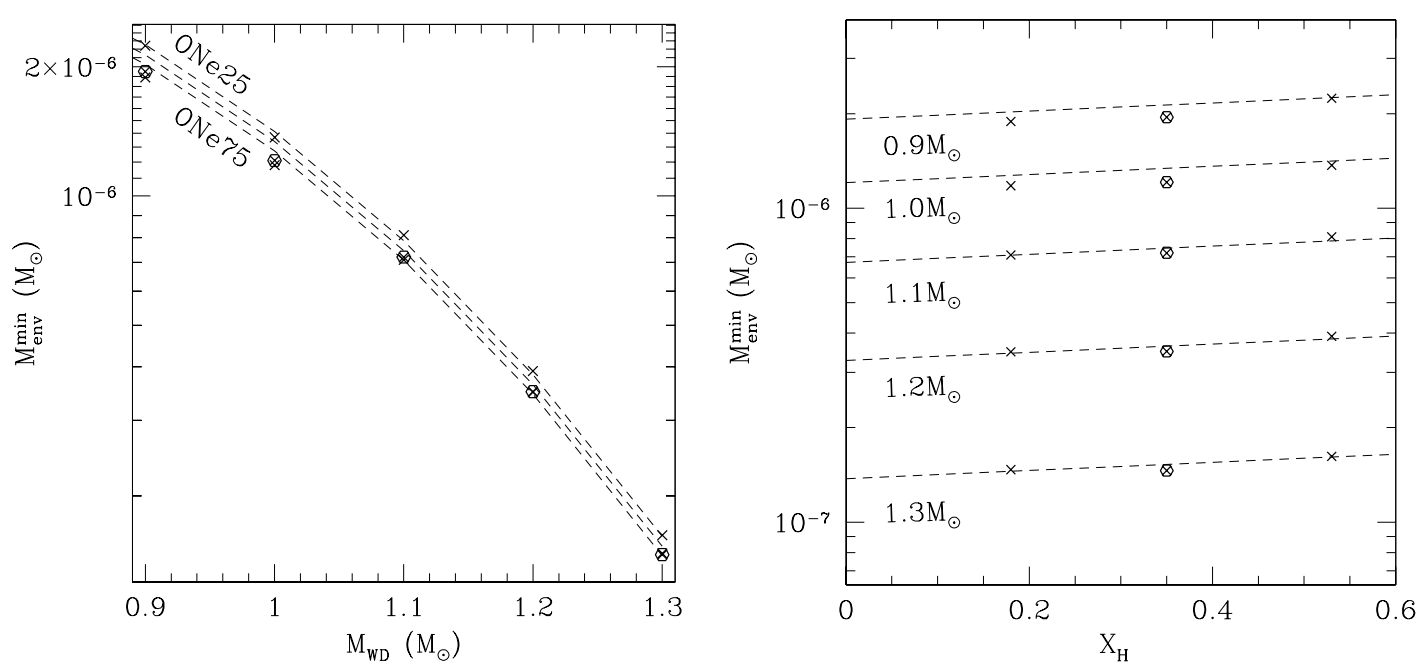

Fig. 9. Minimum envelope mass versus total mass (left) and hydrogen mass fraction (right) for the same models shown in Fig. 6. The minimum envelope mass only slightly depends on the hydrogen mass fraction, resulting in very similar values for models with different compositions (overplotted points for ONe75, ONe50, and CO50 models in left panel). Dashed lines correspond to Eq. (4). 

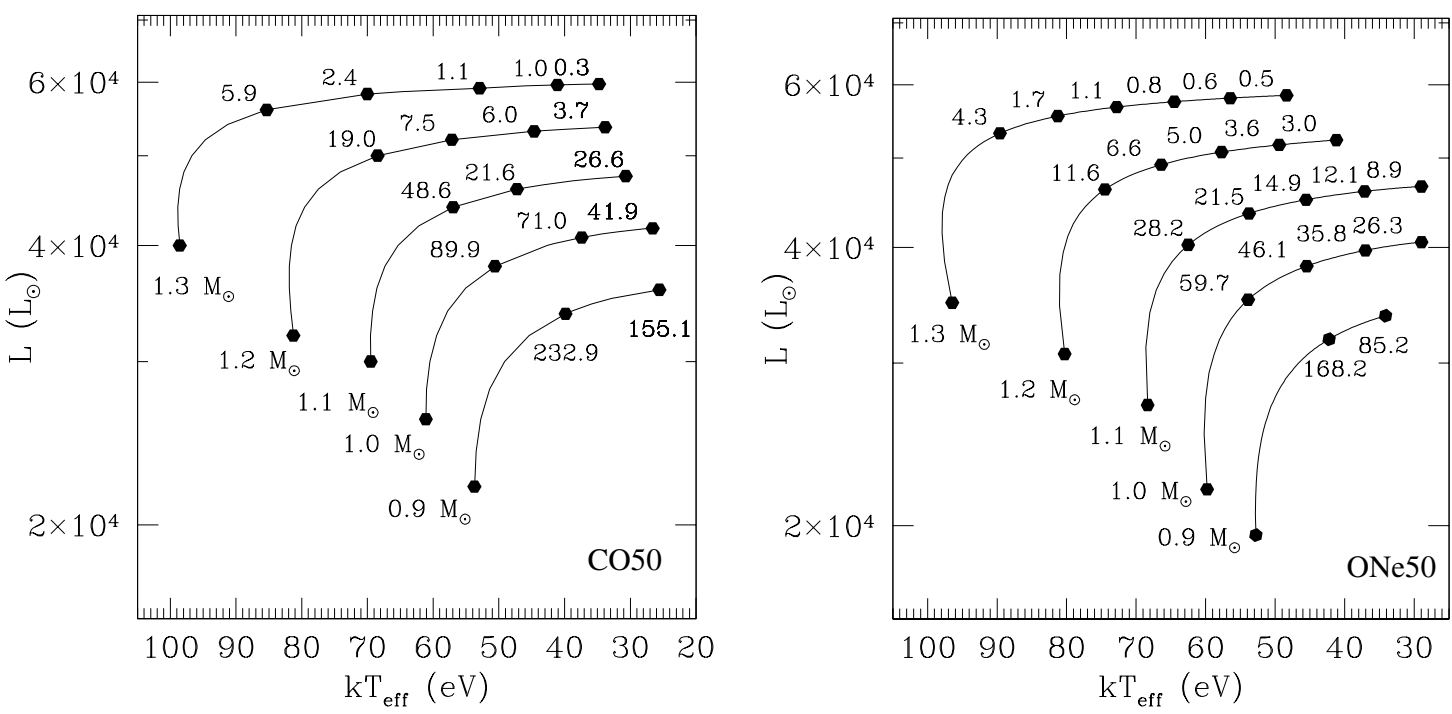

Fig. 10. Luminosity versus effective temperature for the high luminosity branch of CO50 and ONe50 envelopes. Time in days needed for the envelope to evolve between two adjacent ticks is indicated.

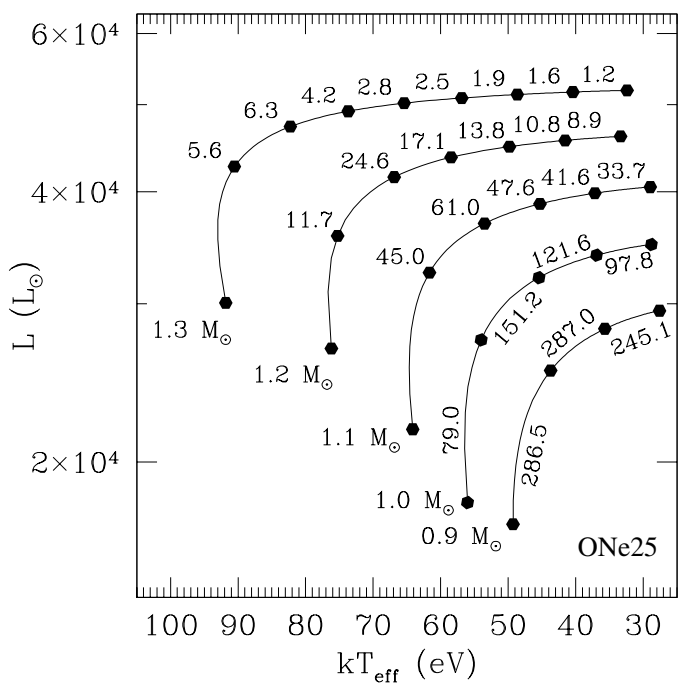

Fig. 11. Same as Fig. 10 for ONe25 and ONe75 envelope models.

for an envelope of $10^{-5}-10^{-4} M_{\odot}$ is decades or even centuries. According to hydrodynamical simulations, $10^{-5}-10^{-4} M_{\odot}$ is the mass required for the thermonuclear runaway to be triggered (José \& Hernanz 1998; Starrfield 1998; Prialnik \& Kovetz 1995), and the fraction of the accreted mass that is expected to be ejected is not large enough to reduce the mass of the remnant by two orders of magnitude. But in the present work, stable envelopes have been found to have masses of $\sim 10^{-6} M_{\odot}$, which requires an extra mass loss mechanism after the nova outburst for the envelope to reach the equilibrium configuration. Once the envelope has reached the steady hydrogen burning phase, the nuclear lifetime can be rewritten with our typical values $\left(M_{\text {env }}=10^{-6} M_{\odot}, L=4 \times 10^{4} L_{\odot}\right)$ as

$\tau_{\text {nuc }}=2 X_{\mathrm{H}}\left(\frac{M_{\text {env }}}{10^{-6} M_{\odot}}\right)\left(\frac{L}{4 \times 10^{4} L_{\odot}}\right)^{-1} \mathrm{yr}$.

For $X=0.35$ (as in ONe50 and CO50 models), $\tau_{\text {nuc }}=$ $0.7 \mathrm{yr} \simeq 255$ days, which agrees with the times in Fig. 10 .

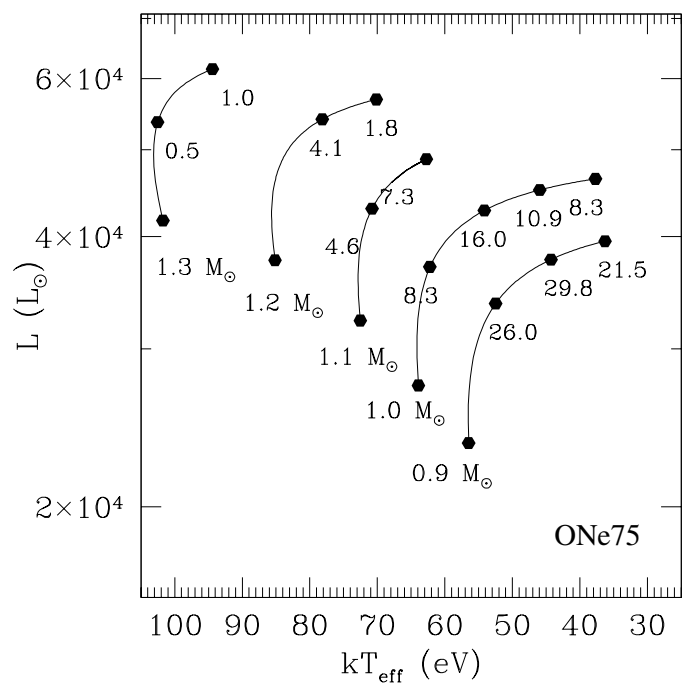

An indication of the typical evolutionary times for our models is given in the last column in Table 2, which lists the time needed for the envelope to change its effective temperature the last $\sim 10 \mathrm{eV}$ before reaching the maximum effective temperature.

\section{Summary}

A numerical model was developed to simulate the physical conditions in the steady hydrogen-burning envelope of a white dwarf after a nova outburst. A grid of envelope models was computed for five different white dwarf masses from 0.9 to $1.3 M_{\odot}$ and four chemical compositions, corresponding to realistic $\mathrm{CO}$ and $\mathrm{ONe}$ white dwarf envelopes with several degrees of mixing between the core material and the solar-like accreted matter. The results show that there exists both a maximum luminosity and a maximum effective temperature for each white dwarf mass and composition. The maximum effective temperature divides the series of models into two branches: a stable 
branch of high, quasi-constant luminosity and a low-luminosity unstable branch with constant photospheric radius. For each white dwarf mass and composition there is also a minimum envelope mass, which occurs in the vicinity of the maximum effective temperature. The mass of the stable branch envelopes is almost constant, decreasing only slowly for increasing effective temperatures. The plateau luminosity, maximum effective temperature, and maximum and minimum envelope masses for each model are summarized in Table 2, as well as the typical evolutionary timescales. Evolutionary timescales are longer for less massive white dwarfs, since luminosity is lower in these cases. The evolution also proceeds more slowly for envelopes with increasing hydrogen abundances, for the same reason.

Each of the overall properties of the envelopes is related to the total white dwarf mass and its chemical composition, and we have found analytical approximations for our ONe envelope models (Eqs. (1)-(4)). Our results show that masses of stable envelopes with steady hydrogen burning $\left(\sim 10^{-6} M_{\odot}\right)$ are in general smaller by at least one order of magnitude than envelope masses needed to trigger the outburst $\left(\sim 10^{-5} M_{\odot}\right)$, according to hydrodynamical models. Since those models predict that only a fraction of the accreted envelope is ejected, it is clear that some mass loss mechanism must act after the outburst, before the steady hydrogen burning can be established in the envelope. An interesting possibility for the mass loss is, as pointed out by Tuchman \& Truran (1998), the dynamical instabilities of the envelope itself when it is left after the outburst with a mass much larger than that of stable configurations.

With the masses found for stable envelopes, the nuclear time-scales are about some weeks or months, similar to observed for post-outburst classical novae. For accreted envelopes highly mixed with the degenerate core, the short duration of the steady hydrogen-burning phase could explain the lack of soft X-ray emission in most novae observed, even if not all the accreted envelope is ejected by the outburst. For those cases where the soft X-ray emission was detected, the photospheric properties obtained with the envelope models and their temporal evolution can be directly compared to the observational results. Comparison of observations to the present models was performed in Sala \& Hernanz (2005, companion paper) for the ROSAT observations of V1974 Cyg. From this comparison, the white dwarf mass, and envelope mass and composition have been constrained.

The present envelope models represent a powerful tool for the study of classical novae, especially if their post-outburst phases are monitored in X-rays. With short and frequent observations that have a good sensitivity in the soft X-ray range and a moderate spectral resolution, the comparison with our envelope models makes it possible to determine the main parameters of the white dwarf. Our envelope models can also be applied to the study of other systems with stationary hydrogen burning on top of a white dwarf, such as supersoft X-ray sources or symbiotic stars.

Acknowledgements. This research was partially funded by the MEC project AYA2004-06290-C02-01. G.S. acknowledges an FPI grant from the MEC.

\section{References}

Böhm-Vitense, E. 1958, Zs. f. Ap., 46, 108

Cash, J. R., \& Karp, A. H. 1990, ACM Trans. Math. Software, 16,201

Cassatella, A., Lamers, H. J. G. L. M., Rossi, C., Altamore, A., \& Gonzalez-Riestra, R. 2004, A\&A, 420, 571

Fontaine, G., \& Van Horn, H. M. 1976, ApJS, 31, 467

Fujimoto, M. Y. 1982, ApJ, 257, 767

Gallagher, J. S., \& Code, A. D. 1973, BAAS, 5, 17

Gehrz, R. D., Truran, J. W., Williams, R. E., \& Starrfield, S. 1998, PASP, 110,3

González-Riestra, R., Orio, M., \& Gallagher, J. 1998, A\&AS, 129, 23

Hamada, T., \& Salpeter, E. E. 1961, ApJ, 264, 282

Henyey, L., \& L'Ecuyer, J. 1969, ApJ, 156, 549

Henyey, L., Vardya, M. S., \& Bondenheimer, P. 1965, ApJ, 142, 841

Iben, I., Jr. 1982, ApJ, 259, 244

Iben. I., Jr., \& Tutukov, A. V. 1989, ApJ, 342, 430

Iben, I., Jr., \& Tutukov, A. V. 1996, ApJS, 105, 145

Iglesias, C. A., \& Rogers, F. J. 1996, ApJ, 464, 943

José, J., \& Hernanz, M. 1998, ApJ, 494, 680

Kato, M., \& Hachisu, I. 1994, ApJ, 437, 802

Kippenhahn, R. 1981, A\&A, 102, 293

Kippenhahn, R., \& Weigert, A., Stellar Structure and Evolution (Berlin: Springer-Verlag), 1990

Krautter, J., \& Williams, R. E. 1989, ApJ, 341, 968

Latour, J. 1970, A\&A, 9, 277

MacDonald, J. 1983, ApJ, 267, 732

MacDonald, J., \& Vennes, S. 1991, ApJ, 373, L51

MacDonald, J., Fujimoto, M. Y., \& Truran, J. 1985, ApJ, 294, 263

Marigo, P. 2000, A\&A, 360, 617

Mihalas, D. 1965, ApJ, 141, 564

Ögelman, H., Beuerman, K., \& Krautter, J. 1984, ApJ, 287, L31

Orio, M., Covington, J., \& Ögelman, H. 2001, A\&A, 373, 542

Paczyński, B. 1970, Acta Astr., 20, 47

Paczyński, B. 1983, ApJ, 264, 282

Press, W. H., Teukolsky, S. A., Vetterling, W. T., \& Flannery, B. P. 1992, Numerical Recipes in Fortran (Cambridge University Press)

Prialnik, D., \& Kovetz, A. 1995, ApJ, 445, 789

Reeves, Stars and Stellar Systems III, ed. L. H. Aller, \& D. B. McLaughin (Chicago: Chicago University Press), 1965

Ritossa, C., Garcia-Berro, E., \& Iben, I., Jr. 1996, ApJ, 460, 489

Sala, G., \& Hernanz, M. 2005, A\&A, 439, 1057

Shore, S. 2002, in Classical Nova Explosions, ed. M. Hernanz, \& J. José, AIP Conf. Proc., 637, 175

Shore, S. N., Sonneborn, G., Starrfield, S., Gonzalez-Riestra, R., \& Ake, T. B. 1993, AJ, 106, 2408

Shore, S. N., Starrfield, S., \& Sonneborn, G. 1996, ApJ, 463, L21

Shore, S. N., Schwarz, G., Bond, H. E., et al. 2003, AJ, 125, 1507

Starrfield, S. 1989, in Classical Novae (New York: Wiley), 39

Starrfield, S. 2002, in Classical Nova Explosions, ed. M. Hernanz, \& J. José, AIP Conf. Proc., 637, 89

Starrfield, S., Sparks, W. M., \& Truran, J. W. 1974, ApJS, 28, 247

Starrfield, S., Truran, J. W., Wiescher, M. C., \& Sparks, W. M. 1998, MNRAS, 296, 502

Tuchman, Y., \& Truran, J. W. 1998, ApJ, 503, 381

Tuchman, Y., Glasner, A., \& Barkat, Z. 1983, ApJ, 268, 356

Vanlandingham, K. M., Schwarz, G. J., Shore, S. N., \& Starrfield, S. 2001, ApJ, 121, 1126 